\title{
SINGLE PHASED BURST ERROR CORRECTING ARRAY CODES
}

\author{
Masahiro Sayano, Rodney M. Goodman and Robert J. McEliece \\ Department of Electrical Engineering \\ California Institute of Technology \\ Mail Code 116-81 \\ Pasadena, California 91125
}

\section{Abstract}

Array codes composed of row and column parities with a diagonally cyclic readout order are capable of correcting a single burst error along one diagonal. A new equation which defines permissible array sizes is presented. These codes have an optimal size which is shown to be a number theoretic problem. In addition, correction of approximate errors is presented; this can be generalized for many classes of error correcting codes.

\section{Size Limits}

An array code was introduced previously to correct phased burst errors. These codes are capable of correcting single phased burst errors and have the added benefits of high speed and effciency. The code is constructed of a two-dimensional array of cells with row and column parity checks, including a check on checks to make the array a complete rectangle. Readout order is along diagonals taken cyclically [1][2][3].

These codes were also shown to have only certain sizes de termined by the array dimensions through a geometric argument In particular, the code was shown to correct a single phased burst given a shorter dimension $n_{1}, n_{2}>n_{1}$, for all $n_{2} \geq 2 n_{1}$; in the range $n_{1}>n_{2}>2 n_{1}$, the code can correct a phased burst if and only if

$$
n_{2} \neq \frac{\alpha+1}{\alpha}\left(n_{1}-\beta\right)
$$

for all $\alpha \geq 1$ and $\beta \geq 1$ [4].

An algebraic approach provides another equation describing this same limitation: Given $n_{2}$, the largest value of $n_{1}$ which will still yield a code capable of correcting errors along a single diagonal of length $n_{1}$ is

$$
n_{1} \leq n_{2}\left(1-\frac{1}{p}\right)
$$

where $p$ is the smallest prime factor of $n_{2}$. This condition is necessary and sufficient.

\section{Optimal Sizes}

From (1) and (2), it is easily shown that the Reiger bound can be met with equality when $n_{2}$ is prime. In this case, $n_{1}=n_{2}-1$, and the code is capable of exceeding the speed while matching the error correcting performance of single error correcting ReedSolomon codes of length $n_{2}$ with $n_{1}$ bits per symbol. Given a fixed $n_{1}$, however, finding the best (smallest) $n_{2}$ is a much more difficult problem. It can be shown that if there exists a prime between $n_{1}$ and $n_{1}+\sqrt{n_{1}}$, then for all $n_{1}$, the best $n_{2}$ is the smallest prime number which is larger than $n_{1}$. This has been confirmed experimentally for all numbers $n_{2}<10,000,000$; since most applications require codewords of much smaller dimensions, this is true for all practical cases. To prove this in general, however, is a nontrivial and unproven number theoretic problem [5][6].

\section{Approximate Errors}

In some applications involving $q$-ary symbols, the most common error is not one where any random value is substituted for the actual one; rather, the erroneous value is often close to the true value. These types of errors will be called approximate errors here. Applications of these errors include high speed modems, multi-valued random access memories, and analog signal coding.

Phased burst approximate errors can be corrected easily with array codes. If the error is assumed to be no more than $\Delta$ away from the true value, then by taking the horizontal parities modulo $2 \Delta+1$ and the vertical parities modulo $\Delta+1$, these errors can be corrected. These parity values can be combined-i.e. weighted and added to use fewer symbols - to obtain codes with yet higher rates.

This concept of taking some parity cells modulo $\Delta+1$ and others modulo $2 \Delta+1$ and packing them into fewer symbols can be generalized to other codes. These will yield higher rate codes without the complexities of bit-sliced coding, another strategy by which approximate errors can be corrected.

\section{Conclusion}

We have presented a new equation describing size limitations of phased burst error correcting array codes. The optimal code size has a longer side which is the smallest prime which is larger than the shorter dimension for all practical applications; to prove this in general is a number theoretic problem. In addition, a means to correct approximate errors has been presented; this approach can be generalized for other codes.

\section{References}

[1] P. G. Farrell and S. J. Hopkins, "Burst-Error-Correcting Array Codes," The Radio and Electronic Engineer, vol. 52, pp. 188192, 1982.

[2] M. Blaum, "Error-Correcting Codes for Computer Memories," Ph.D. Thesis, California Institute of Technology, 1985.

[3] M. Blaum, P. G. Farrell, and H. C. A. van Tilborg, "A Class of Burst Error-Correcting Array Codes," IEEE Transactions on Information Theory, vol. IT-32, pp. 836-839, 1986.

[4] R. M. Goodman and M. Sayano, "Size Limits on Phased Burst Error Correcting Array Codes," Electronics Letters, vol. 26, pp. 55-56, 1990.

[5] G. H. Hardy and E. M. Wright, An Introduction to the Theory of Numbers, Oxford University Press, 1979.

[6] P. Ribenboim, The Book of Prime Number Records, SpringerVerlag, 1989. 